\title{
Evaluation of Phenolic Compounds, Antioxidant and Antimicrobial Activities of Rice (Oriza Sativa. L.) Pedicle extract (In Vitro)
}

\author{
Bahar Ahmadi, Aazam Aarabi* \\ Department of Food Science and Technology, Shahreza Branch, Islamic Azad University, Shahreza, Iran \\ Email: aarabi@iaush.ac.ir (Corresponding author)
}

Received: 27 October 2018; Accepted: 15 November 2018; Available online: 15 February 2019

\begin{abstract}
In this study, rice pedicle extracts (Oriza sativa. L.) were obtained by high pressure reactor using water, and sodium hydroxide at temperatures of 70, 90, $110130{ }^{\circ} \mathrm{C}$. The extracts were evaluated for total phenolic content, antioxidant activity and growth inhibition of Escherichia coli and Candida albicans. Their results were compared to Soxhlet extraction by using ethanol $(95 \% \mathrm{v} / \mathrm{v})$. The results showed that the use of different temperatures had a significant effect on the percentage of phenolic compounds, and the alkali method had a significant effect on the amount of phenolic compounds rather than water extraction (128.72 and 51.79 GA/g, respectively). The highest antimicrobial activity levels were obtained in ethanol extracts that the extracts have inhibitory effect on $E$. coli. None of extracts had inhibitory effect on Candida albicans. The results provided the evidence that the studied rice (Oriza sativa. L.) pedicle extract might be potential sources of phenolic compound, natural antioxidant and antimicrobial agents.
\end{abstract}

Keywords: Escherichia coli; Oriza sativa. L.; Phenolic compounds; Pedicle.

\section{Introduction}

Fat oxidation is one of the main economic concerns for food products. Fat oxidation in food sharply reduces their maintenance, making food quality unacceptable to the customer. Antioxidants are substances that may be inherent in the product, or it might be added to food through a process. The use of substances increasing food quality by delaying lipid oxidation has been experienced for centuries. Antioxidants are compounds maintaining food and reducing the speed and intensity of chemical reactions by slowing the corruption, keen or discoloration due to oxidation [1].

A large number of natural and synthetic antioxidant compounds are used in the treatment or prevention of multiple diseases. Due to adverse effects of synthetic antioxidants in the body, now greater effort has been made to replace them with natural products, especially agricultural products containing natural antioxidants [2] . Phenolic compounds are one of the main groups preventing diseases such as atherosclerosis and cancer. Applying natural phenolic acids as compounds involving antioxidants has attracted researchers' attention[3]. In addition, many phenolic compounds has been reported as strong antioxidants compounds[4]. Furthermore, by neutralizing the free radicals, antioxidants can reduce the risk of cardiovascular disease, and block cancer progression.

Nowadays, grain provides more than half of the needed food of the world population. Furthermore, rice with the scientific name of Oriza sativa. L. is a plant with various compounds such as carbohydrates, fat, protein, fiber, ash and minerals, playing an important role in meeting the needs of the human body [5]. After wheat, rice is one of the most important grains used by people of the world, but compared to wheat, it has far less industrial value. Instead, consumption of rice for eating is highly important. In rice bran, a remarkable combination of approximately 500 (ppm) of Tocotrienol has been known belonging to the family of vitamin E categorized as powerful natural antioxidants [6]. Studies showed that physical effect of rice bran oil was due to Gamma -Oryzanol that its high amount in rice bran oil was significant. Moreover, the Oryzanol present in rice bran oil has antioxidant activity to prevent oxidation 6 times more than $\alpha$-Tocopherol. Now when harvesting rice, its pedicle is a by-product of limited use and is used only for animal feed. Given that many studies are not seen in the field of extraction of bioactive and useful compounds of rice pedicle. To produce added value material, this study aimed to evaluate total phenolic compounds and antioxidant activity of extracts obtained from rice pedicle in aqueous and alkaline media in high pressured- reactor.

\section{Materials and methods}




\subsection{Materials}

All chemicals used in this study, including sodium hydroxide $(\mathrm{NaOH})$, acetic acid, potassium Ferrocyanide, Zinc acetate (as carez reagents 1 and 2), hydrochloric acid, ethanol, and methanol were purchased from German Merck Co, and the nutrient Broth media was purchased from Biofield Co.

\subsection{Apparatus}

In the present study, high-pressured reactor (The autoclave used in this study has a cylindrical jacketed body with a capacity of $1.5 \mathrm{~L}$, diameter of $15 \mathrm{~cm}$ and a height of $66 \mathrm{~cm}$. Haake thermostatic bath (model N2-B, Karlsruhe, Germany) in the reactor was used heat and control the temperature. The temperatures inside the reactor and in the thermostatic bath were measured using thermocouples type K (nickel-chromium/nickel-aluminum), digital scales (methrom), rotary vacuum evaporators (Heidolph), spectrophotometer (Perkin Elmer), centrifuges (Sigma, Germany) autoclaves and sampler.

\subsection{Plant material}

Rice pedicles were purchased from one of the rice farms in Isfahan and ground in a laboratory mill (Panasonic MX -J120-P made in Japan). The powdered sample was sieved with mesh size $1 \mathrm{~mm}$ and, the samples were wrapped in nylon at ambient temperature.

\subsection{Extraction procedure}

\subsubsection{Extraction with under pressure water}

For extraction, $20 \mathrm{~g}$ of rice pedicle powder was mixed with 1 liter of distilled water, and then the mixture was transferred to the reactor system and maintained at 70, 90, 110 and $130^{\circ} \mathrm{C}$ for 45 minutes. After this time, the temperature decrease operation to $20^{\circ} \mathrm{C}$ was carried out by the reactor cooling system (Figure 1 ). To remove excess water, the under vacuum evaporator at a temperature of $50^{\circ} \mathrm{C}$ was used, and the remaining solution was transferred to a small jar with a Teflon lid and kept in the $-18 \mathrm{c}^{\circ}$ freezer for testing.
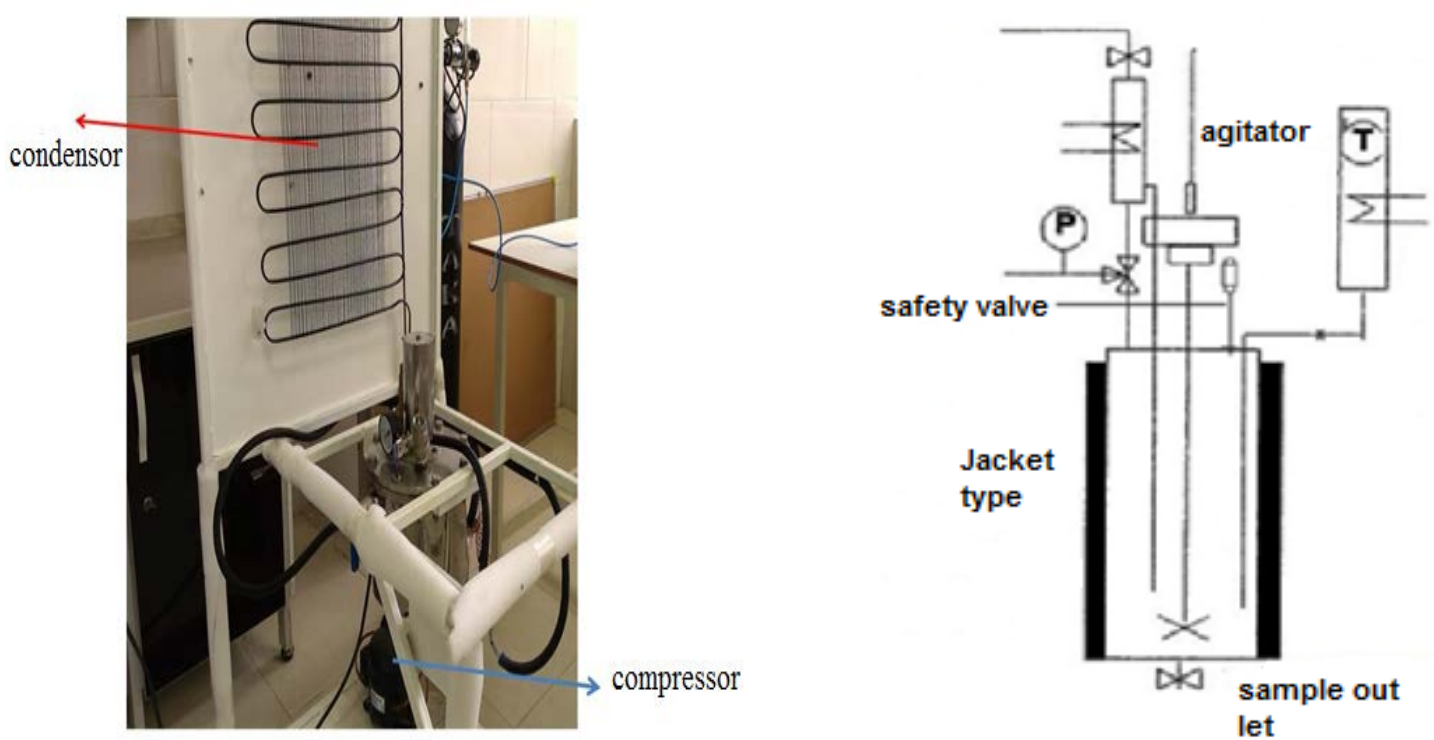

Figure 1. Picture of pressure reactor vessel with cooling system

\subsubsection{Extraction with alkali under pressure}

In this method, $20 \mathrm{~g}$ of rice pedicle were mixed in $1 \mathrm{~L}$ of $\mathrm{NaOH}(1 \mathrm{~mol} / \mathrm{L})$ and transferred to the reactor, kept at the temperatures of $70,90,110,130^{\circ} \mathrm{C}$ for 45 minutes to perform hydrolysis reactions and destroy tissue rice pedicle. After this time, the reactor contents were collected to remove impurities and deposition of cellulosic materials using hydrochloric acid 2.5 molar, and $\mathrm{pH}$ of solution was reduced to $\mathrm{pH}=7$. Afterwards, the process of smoothing solution with filter paper was carried out. $50 \mathrm{ml}$ of the filtered solution was mixed with 95\% ethanol, and centrifuged at $3000 \mathrm{rpm}$. The supernatant was transferred to vacuum rotary evaporator to increase extract concentration. The extracts were used to measure the total phenol and antioxidants compounds.

\subsubsection{Ethanol extraction using Soxhlet}


For the Soxhlet method, $2 \mathrm{~g}$ of rice pedicle were weighted and mixed with $100 \mathrm{ml}$ of $95 \%$ ethanol and transferred to the Soxhlet and the temperature was set to $70^{\circ} \mathrm{C}$, and it was kept for 45 minutes at this temperature, and extraction operation was performed.

\subsection{Determination of total phenol compounds (TPC)}

The total amount of phenolic compounds (TPC) in alkaline and aqueous extract was measured based on FolinCiocalteu. $200 \mathrm{mg}$ of the extract was added to $1000 \mu \mathrm{L}$ of Folin-Ciocalteu and $3 \mathrm{~mL}$ of sodium carbonate $(20 \% \mathrm{~W} / \mathrm{V})$, and the solution was placed in a dark room for 30 minutes to complete the required reactions, and absorbance was read at $760 \mathrm{~nm}$ by spectrophotometer. The TPC was expressed as $\mathrm{mg}$ Gallic acid per gram of dry matter [7].

\subsection{Antioxidant activity evaluation}

Anti-radical activity was determined by DPPH test. A fixed volume $(0.1 \mathrm{ml})$ of water, alcoholic and alkaline extracts was added to the DPPH solution ( $3.9 \mathrm{ml}$ of $0.3 \mathrm{mM}$ solution of DPPH). After 1 hour, absorbance of the solution was measured by spectrophotometer at $517 \mathrm{~nm}$. Anti-radical activity was calculated using equation (1)[8] .

Antioxidant activity $=\frac{A 1-A 0}{A 0} \times 100$

In this regard $\mathrm{A}_{0}$ : absorption in control sample, $\mathrm{A}_{1}$ : absorption in the samples.

\subsection{Antimicrobial activity}

Antimicrobial activity of aqueous, alcohol and alkaline extracts on E. coli $\mathrm{O}_{157} \mathrm{H}_{7}$ (ATCC25932) and Candida albicans obtained from the Iranian Research Organization for Science and Technology was examined.

\subsubsection{Preparation of inoculum}

Bacterial strains were cultured and inoculated at $37^{\circ} \mathrm{C}$ overnight in Nutrient Broth media (NB) and used as inoculum. The turbidity of the inoculum was adjusted to $2 \times 10^{8}$ (cells $/ \mathrm{ml}$ ) in comparison to McFarland turbidity.

\subsubsection{Antibacterial activity}

Agar-well diffusion method was performed to evaluate antibacterial activity [9]. The bacterial inoculum was evenly spread onto the surface of the nutrient agar plates using sterile swab sticks.

Agar plates were prepared using sterile nutrient agar and yeast extract glucose chloramphenicol agar for EColi and Candida albicans. Wells (5 mm diameter) were punched in the plates using a sterile stainless steel borer. 10 $\mu \mathrm{l}$ of each extract concentrations were added to each well, and incubated at $37^{\circ} \mathrm{C}$ (for EColi) and $25^{\circ} \mathrm{C}$ (for fungi) for $24 \mathrm{~h}$.The plates were observed for the presence of a clear zone around the wells. The absence of a zone inhibition was interpreted as the absence of activity, and the size of the clear zones around the wells was measured, and the antibacterial activity was expressed in terms of the average diameter of the zone inhibition in millimeters [10]. Each experiment was tested in triplicate.

\subsection{Data analysis}

This study was conducted in the form of a factorial experiment in a completely randomized design with three replications. The averages were compared by Duncan and at the significant level of 0.05 . The SPSS software version 17 was used for analysis of variance and comparison of means, and the Excel -2007 software was used to draw the graphs.

\section{Results and discussion}

\subsection{Measurement of total phenols}

The results showed a significant difference in the amount of extracted phenolic compounds. In addition, using different temperatures to extract had a significant effect on increase percent of the phenolic compounds, therefore, by increasing temperature from 70 to $130^{\circ} \mathrm{C}$, the phenolic compounds had a significant increase. (Table 1 and Figure. 2).

At a constant temperature of $70^{\circ} \mathrm{C}$, the maximum amount of phenol was observed in the extraction of alkali (sodium hydroxide), then ethanol and finally water extraction. In various reports, it is mentioned that generally alcoholic solvents are used for phenol extraction from natural resources. Ethanol and methanol with water are more capable in extracting phenolic compounds. Due to decrease in polarity compared to pure solvents, alcoholwater mixture has the solubility capability of phenolic compounds with lower polarity and increases the extraction rate of phenolic compounds several times[11]. Considering the issues, the reason of high rates of phenolic 
compounds extraction in ethanol extract rather than water can be justified. In addition, reviewing the results indicated that although in the aqueous method, by increasing temperature, phenolic compounds also increased in comparison to the alkaline method, releasing and extracting phenolic compounds were less. Comparing the results of the ethanol and alkaline methods, it can be mentioned that ethanol is not able to penetrate into cell all by itself, and does not have the same ability as alkaline to hydrolyze complex lignin-ferulic acid-polysaccharide, as a result, it can extract only the phenolic compounds as the result of heating and lignin destruction.

Table 1. The total amount of phenolic compounds mg of Gallic acid per gram of extract, extracts from rice pedicle, temperature $\left({ }^{\circ} \mathrm{C}\right)$

\begin{tabular}{lcccc}
\hline solution & $\mathbf{7 0}$ & $\mathbf{9 0}$ & $\mathbf{1 1 0}$ & $\mathbf{1 3 0}$ \\
\hline Water & $37.73 \pm 0.17^{\mathrm{Aa}}$ & $42.08 \pm 0.32^{\mathrm{Aa}}$ & $42.98 \pm 0.1^{\mathrm{Aa}}$ & $111.22 \pm 0.11^{\mathrm{Aa}}$ \\
$\mathrm{NaOH}$ & $116.23 \pm 0.15^{\mathrm{Cc}}$ & $121.06 \pm 0.3^{\mathrm{Bb}}$ & $124.04 \pm 0.05^{\mathrm{Bb}}$ & $128.72 \pm 0.17^{\mathrm{ABb}}$ \\
ethanol & $74.50 \pm 0.6^{\mathrm{Bb}}$ & - & - & - \\
\hline
\end{tabular}

\pm Values represent the standard deviation.

(a-d): Averages having different superscripts in a row have a significant difference (less than 0.05).

(A-C): Averages having different superscripts with capital letters in a column differ significantly (less than 0.05) (statistical difference between treatments).

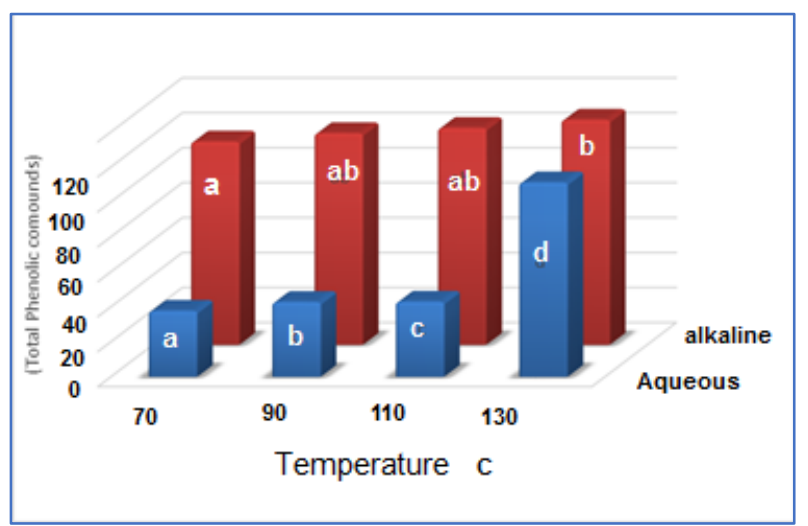

Figure 2. The effect of solvent types on the extraction of phenolic compounds (mg Gallic acid/g extract)

Moreover, in examining various levels of temperature, temperature of $130^{\circ} \mathrm{C}$ had the highest phenolic compounds than other applied temperatures for extraction. The phenolic compounds derived from rice pedicle extracts in all three methods have significant differences with each other in the test area. Due to the high amount of total phenol at a temperature of $130^{\circ} \mathrm{C}$ compared to other temperatures used in both media, it can be interpreted that the temperature can increase rupture of cells in most plants and destroy polysaccharides such as pectin. Accordingly, it can release phenol compounds connected to the pectin available in cell wall components [12] As a polar solvent, water can naturally extract polar compounds better than non-polar ones. High temperatures reduce solvent polarity, thereby improving the ability to dissolve compounds with low polarity. Temperature increase can reduce surface tension and viscosity of the solvent. It also increases the velocity and mass transfer rate during extraction [13]. Scalbert et al. found that the presence of phenolic acids in wheat pedicle cause the bridge and the formation of a complex set of polysaccharide -ester- ferulic acid- ether -lignin. In addition, alkali treatments cause lignin saponification followed by dissolution and break of ester joints in the complex of lignin - polysaccharide[14]. Consequently, acidic compounds and aromatic aldehydes such as hydroxy Benz aldehyde, vanillin and molecules associated with lignin precursors are produced, among which vanillin and Ferulic acid are the most important products[15]. Thus, increasing the concentration of phenolic compounds in a hydrolyzed solution by alkaline can indicate that a large number of joints between ferulic acid and lignin and polysaccharides are of the ester type. The use of alkali, particularly sodium hydroxide causes hydrolysis of lignin and improves performance of the aromatic ring in fragmentation of lignin. Thus, it can be mentioned that using alkali media to break the lignin to release phenolic compounds acts better than the water media, and the release of phenolic compounds is higher using alkaline medium rather than water [16].

\subsection{Anti-radical activity}

Radical inhibitory test of DPPH provides basic information in the field of anti-radical activity of the herbal phenolic extract. As this method requires a shorter time rather than other methods, therefore, it is widely applied to evaluate anti-oxidant activities [17]. Between the three extraction methods and the used temperatures, there is a 
significant difference at the level of $\mathrm{p}<0.05$. Anti-radical activity in both extracts (aqueous and alkaline) depended on the media and extraction environment, and the antioxidant power in the alkaline extract was more than aqueous extraction (Figure 3). Certainly, as it is observed in the results (Table 2), in both alkaline and aqueous methods, by increasing the extraction temperature in the temporal range of 90-130 ${ }^{\circ} \mathrm{C}$, inhibiting power of free radical represented a fixed process along with a decrease in anti-oxidant activity. It can be stated that among the studied temperatures to take out the extract, the highest and lowest amounts of anti-radical activity were attributed to 70 and $130^{\circ} \mathrm{C}$, respectively (111.22 and 37.73) in aqueous extraction, and in alkaline extraction, they were 70 and $130^{\circ} \mathrm{C}$ (128.82 and 116.23). As Figure 3 shows, the higher inhibitory activity of free radical of DPPH in the alkaline method compare to the aqueous method can be attributed to the phenolic content of the extracts. Totally, the concentration increase of phenolic compounds directly increases the ability of different extracts to inhibit free radicals. As Tables 1 and 2 show, due to the higher phenolic compounds reported as potent antioxidants [4], in the alkaline extraction, the possibility of donating hydrogen to free radicals and subsequent inhibition of the extract increases. These results are consistent with the reports of Bauer et al.

Furthermore, in comparing the results at $70^{\circ} \mathrm{C}$ among the three aqueous, alkali and ethanol extraction methods, the highest amount of inhibitory power was for the ethanol method that is probably due to some compounds that are not in the category of phenol compounds, but they have inhibitory effect of free radical (Figure. 4).

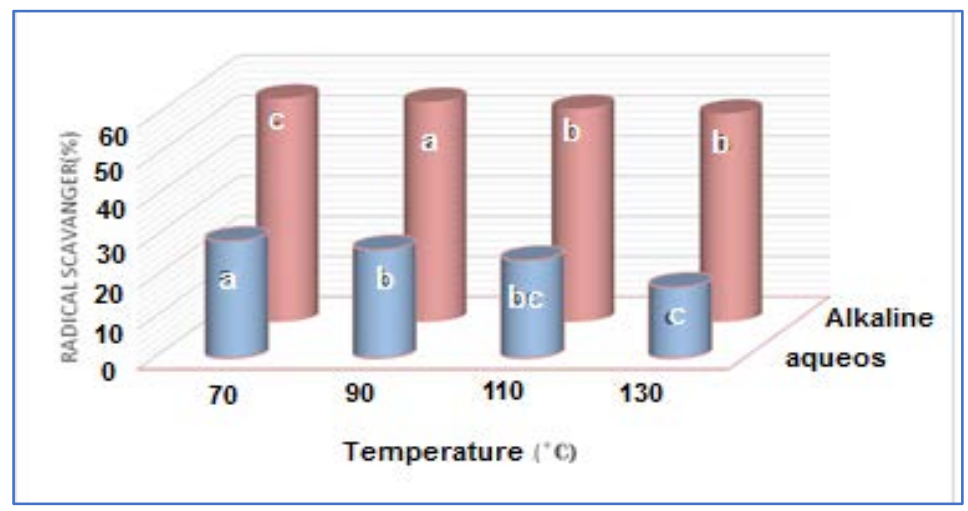

Figure 3: Effect of extraction method on the percentage of DPPH inhibitory activity

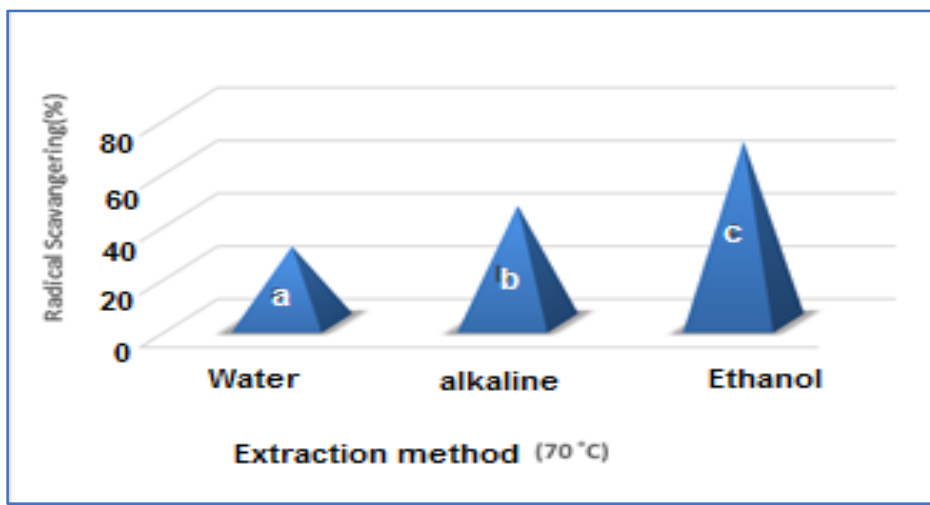

Figure 4: Effect of extraction method on the percentage of DPPH inhibitory activity at $70^{\circ} \mathrm{C}$

\subsection{Antimicrobial test}

The extracts obtained at the temperature of $70^{\circ} \mathrm{C}$ by aqueous, alkali and ethanol methods on Gram-negative bacteria (E. coli) and Candida albicans were studied using the agar well diffusion method (Table 2).

The results indicated that the extracts obtained from two water and ethanol treatments had an inhibitory effect on E. coli (Table 2), and despite higher amount of phenol compounds in the alkaline extract, its inhibitory effect on E.coli bacteria was not observed at this temperature. In addition, it was found that none of extracts showed the ability to prevent Candida albicans.

In comparing aqueous and alkaline methods, it can be concluded that the presence of $E$. coli in ethanol extract increases its sensitivity and makes a larger halo that can be justified by the presence of more phenolic compounds in the ethanol extract. Additionally, phenolic compounds are a broad group of effective compounds available in plant extracts made in response to microbial infection in plants. They are also active against a range of micro- 
organisms, and the antimicrobial effect of phenolic compounds can be due to creation of their complex with outer membrane and soluble proteins attached to the membrane of microorganisms [18]. Furthermore, the compounds cause antimicrobial effect by penetrating into cell membrane and breaking it. Phenol compounds apply their antimicrobial effect by interference in operation of cell membrane and interfering with the entry and exit of substances into the cell [19].

Higher sensitivity of bacteria in ethanol extract rather than alkaline might be due to the multi-layered wall of this bacteria. In other words, Gram-negative bacteria have an outer membrane and peri-plasmic space. Thus, this membrane is known as a barrier to diffusion of multiple antibiotics. Moreover, this membrane prevents penetration of hydrophilic to the bacteria. Peri-plasmic area also includes many enzymes that are able to decompose foreign molecules coming from the outer space [20].

Various studies have shown that Gram-negative bacteria are more resistant to extracts obtained in different ways rather than Gram-positive bacteria. This is owing to the hydrophilic cell wall structure of Gram-negative bacteria such as lipopolysaccharide limiting hydrophobic compounds release into their oils. However, E. coli may be sensitive to ethanol media due to its more sensitivity to the compounds in the extract process of this method, since presence of extracted compounds in this method has probably been effective in permeability of the outer layer of bacteria [21].

Table 2. Comparison of different extraction methods on growth inhibition of E. coli and Candida albicans

\begin{tabular}{rrr}
\hline \multirow{2}{*}{ Type of extraction method } & & Average of Area (cm) \\
\cline { 2 - 3 } Aqueous & E. coli & Candida albicans \\
Alkali & $0.4 \pm 0.01 \mathrm{a}$ & - \\
Ethanol & - & - \\
\end{tabular}

* The numbers in the table on average three times

\pm Values represent the standard deviation. Different letters in each column indicate a significant difference in the level of 5 percent.

\section{Conclusions}

The study results indicated that total phenolic compounds were obtained from three extracting alkaline, ethanol and aqueous methods. Selecting a suitable temperature has a significant effect on the phenol compounds such, that by increasing temperature to $130^{\circ} \mathrm{C}$, the amount of phenol compounds and inhibitory power increased significantly at the studied level. The results of the extraction methods with different solutions indicate the effect of type of solution on the extracted phenol compounds. In addition, separation and identification of phenol compounds of rice pedicle using HPLC are recommended.

\section{References}

[1] Dimitrios B. Sources of natural phenolic antioxidants. Trends in Food Science \& Technology. 2006;17:505512.

[2] Singh G, Marimuthu, P, De Heluani, C. S and . Catalan, C. A. N. Antioxidant and Biocidal Activities of Carum nigrum (Seed) Essential Oil, Oleoresin, and Their Selected Components. J Agric Food Chem. 2006; 54 (1): 174-181.

[3] Kahl R, Kappus, H. Toxicology of the synthetic antioxidants BHA and BHT in comparison with the natural antioxidant vitamin E. Z Lebensm Unters Forsch. 1993;196(4):329-338.

[4] Serrano J, Goñi I, Saura-Calixto F. Food antioxidant capacity determined by chemical methods may underestimate the physiological antioxidant capacity. Food Research International. 2007;40(1):15-21.

[5] Amissah JGNE, W.O. Oduro, I. Manful, J.T. Nutrient composition of bran from new rice varieties under study in Ghana. Food Control. 2003; 14:21-24.

[6] Nagendra Prasad Mn NP, Kr S, Khatokar M S. Health Benefits of Rice Bran - A Review. Journal of Nutrition \& Food Sciences. 2011; 01(03).

[7] Baba SA, Malik SA. Determination of total phenolic and flavonoid content, antimicrobial and antioxidant activity of a root extract of Arisaema jacquemontii Blume. Journal of Taibah University for Science. 2015; 9(4):449-454. 
[8] Brand-Williams WC, M. E. and Berset, C. . Use of a free radical method to evaluate antioxidant activity. Lebensm-Wiss u-Technol. 1995;28:25-30.

[9] Borrás-Linares I, Fernández-Arroyo S, Arráez-Roman D, Palmeros-Suárez PA, Del Val-Díaz R, AndradeGonzáles I, et al. Characterization of phenolic compounds, anthocyanidin, antioxidant and antimicrobial activity of 25 varieties of Mexican Roselle (Hibiscus sabdariffa). Industrial Crops and Products. 2015; 7(69):385-394.

[10] Ture H, Eroglu E, Ozen B, Soyer F. Effect of biopolymers containing natamycin against Aspergillus niger and Penicillium roquefortii on fresh kashar cheese. International Journal of Food Science \& Technology. 2011; 46(1):154-160.

[11] Ana Bucić-Kojić MP, Srećko Tomas, Stela Jokić, Ibrahim Mujić, Mate Bilić, Darko Velić. Effect of Extraction Conditions on the Extractability of Phenolic Compounds from Lyophilised Fig Fruits (Ficus Carica L.). Pol J Food Nutr Sci. 2011; 61(3):195-199.

[12] Bauer JL, Harbaum-Piayda B, Schwarz K. Phenolic compounds from hydrolyzed and extracted fiber-rich byproducts. LWT - Food Science and Technology. 2012; 47(2):246-254.

[13] Ramos S. Effects of dietary flavonoids on apoptotic pathways related to cancer chemoprevention. Journal of Nutritional Biochemistry. 2007; 18(7):427-442.

[14] Scalbert A, Monties, B, Lallemand, J.Y., Guittet, E. and Rolando, C. . Ether linkage between phenolic acids and lignin fractions from wheat straw. Phytochemistry. 1985; 24 (6):1359-1362.

[15] Salleh NHM, Daud MZM, Arbain D, Ahmad MS, Ismail KSK. Optimization of alkaline hydrolysis of paddy straw for ferulic acid extraction. Industrial Crops and Products. 2011; 34(3):1635-1640.

[16] Werhan H. A Process for the Complete Valorization of Lignin into Aromatic Chemicals based on Acidic Oxidation. A dissertation submitted to ETH Zurich for the degree of Doctor of Sciences. 2013, 1-215.

[17] Floegel A, Kim D-O, Chung S-J, Koo SI, Chun OK. Comparison of ABTS/DPPH assays to measure antioxidant capacity in popular antioxidant-rich US foods. Journal of Food Composition and Analysis. 2011; 24(7):1043-1048.

[18] Deshpande SS. Handbook of Food Toxicology. CRC Press; 2002.p. 920

[19] Lee O-H, Lee B-Y. Antioxidant and antimicrobial activities of individual and combined phenolics in Olea europaea leaf extract. Bioresource Technology. 2010; 101(10):3751-3754.

[20] Alañón ME, García-Ruíz A, Díaz-Maroto MC, Pérez-Coello MS, Moreno-Arribas MV. Antimicrobial and antioxidant activity of pressurized liquid extracts from oenological woods. Food Control. 2015; 50:581-588.

[21] Pripdeevech P, Chumpolsri W, Suttiarporn P, Wongpornchai S. The chemical composition and antioxidant activities of basil from Thailand using retention indices and comprehensive two-dimensional gas chromatography. Journal of the Serbian Chemical Society. 2010; 75(11):1503-1513.

(C) 2019 by the author(s). This work is licensed under a Creative Commons Attribution 4.0 International License (http://creativecommons.org/licenses/by/4.0/). Authors retain copyright of their work, with first publication rights granted to Tech Reviews Ltd. 